\title{
Adolescents May Be Older Than We Think: Today 25 Is the New 18, or Is It?
}

\author{
Jacqueline M. Arnone* \\ Kean University, New Jersey, United States \\ *Corresponding author: Jackiea412@aol.com \\ Received May 02, 2014; Revised May 02, 2014; Accepted May 05, 2014
}

\begin{abstract}
There is a marked dearth of research on the lived experience of adolescents with celiac disease in the United States in the current literature. A vulnerable population, adolescents with celiac disease can get lost in the gap between healthcare research and practice related to treatment regimens, health teaching and health counseling. The period of adolescence is triphasic and a time of rapid physical growth and development. It includes developmental tasks of identity formation, the separation from authority as a means of independence and seeking conformity with peers. Varying age range brackets for adolescents were identified through a review of the current literature of adolescents with celiac disease. The lack of universality in age bracketing prompted the question, "What is the age range for the period of adolescence?” Current literature surveyed from the United States and Great Britain state that 25 is the new 18. Social and cultural factors and neuroscience research support that adolescents may be older than we think. In the United States there are more than 80 million Millenials also known as Generation Y, born between 1980 and 1995, that fall within this expanded stage of "emerging adulthood.” The general consensus across disciplines remains undecided in benchmarking an expanded age bracket for adolescence. Further discourse and research is warranted to determine a universal age standard for the period of adolescence, as it relates to the research of Millenial/Generation Y adolescents with celiac disease.
\end{abstract}

Keywords: adolescence, adolescent, celiac disease, adolescence, Generation $Y$

Cite This Article: Jacqueline M. Arnone, “Adolescents May Be Older Than We Think: Today 25 Is the New 18, or Is It?” International Journal of Celiac Disease, vol. 2, no. 2 (2014): 47-48. doi: 10.12691/ijcd-2-2-4.

\section{Introduction}

There is a marked dearth of research on the lived experience of adolescents in the United States in the extant literature [1]. Adolescents, especially those with celiac disease, are a vulnerable population that can get lost in the gap of healthcare research and evidence based practice related to treatment regimens, health teaching and health counseling. In doing preliminary research, it was demonstrated that there are varying age ranges in published studies denoting the age bracket for adolescents. While most cite 13-19 years old, there were some studies that used 18 years old as the end age for this period. Thus, my question became, "What is the age range for the period of adolescence?” While a clear and universal answer could not be found across disciplines, there is current dialogue in the literature asking the same question, and the answer might just surprise you.

\section{The stage of Adolescence}

As scholars, we define our terms, and therefore clear and concise definitions of the terms are required. The word adolescence is derived from the Latin adolescere, which means to grow up. Adolescence is defined as: the transitional period between puberty and adulthood in human development, extending mainly over the teen years, and terminating legally when the age of majority is reached [2]. The word adolescent, is defined as: (of a young person) in the process of developing from a child into an adult [3]. The term "adolescence", first introduced by G. Stanley Hall in 1904, described a "new cultural phenomenon that emerged in the United States and other industrialized societies in the late 19th century" [4,5]. The period of adolescence, according to lifespan developmental psychologists, occurs from age 13 to19 years old, is triphasic and viewed as a time of "storm and stress [6]. Adolescence is the critical bridge between childhood and adulthood. This period marks a time of rapid physical growth and development, and includes tasks of identity formation, the separation from authority as a means of independence, and seeking conformity with peers [6].

\section{The Changing Times of Adolescence}

Historically adolescents have been around since the beginning of time, yet they were not a specifically distinguishable or categorized cohort in many cultures and societies. The attainment of puberty has traditionally been viewed as the harbinger of reaching "maturity". In the past 100 years, societal influences have impacted the benchmarks of maturity and transition to adulthood. Two 
examples are the right to vote, and the increase of the legal age to consume alcoholic beverages [7].

It seems now that twenty-five is the new eighteen. In the past, a 25 year old would be considered to be in the life stage of early adulthood. This is a time for finding intimacy with a partner, marriage and parenting [6]. Times have dramatically changed and so has the age range of adolescence. "Gone is the notion that adulthood officially started at 18, when one graduated from high school- or even 21, the modern-day age limit for drinking alcohol” [8]. In a survey done by the University of Chicago in 2003, Americans concurred with this new "age" denoting maturity, supporting that adulthood starts at 26 years old [8].

\section{Social and Cultural Impact}

Social and cultural factors such as attaining higher levels of education due to the inability to find employment after college, moving home with parents, and delaying marriage, family and house ownership all attribute to this new age span of "emerging adulthood" $[8,9]$. In an interview by Christina Scotti (2014) with Jeffrey Arnett, professor at Clark University, Arnett states the process of this prolonged entrance into adulthood goes beyond the 80 million Millenials in the United States born between 1980 and 1995, noting a gradual paradigm shift of this process over the past two decades.

Health wise compared with other generations, the Millenials aged 18-33 years old are "the most stressed out generation and do not believe they are doing enough to manage their stress" [9]. Technically savvy, this generation displays a deficit in social skills when dealing with problematic circumstances or people and highly favors texting, email and social networking as opposed to talking on the telephone or having face to face conversations [10].

\section{Neuroscience Research}

Additionally, there is other scientific evidence supporting that adolescents may be older than we think. Neuroscience research of adolescent brains also shows the age to adulthood may now extend to 25 years old. Employing imaging technologies such as structural and functional magnetic resonance imaging (sMRI and fMRI), findings demonstrate that.

Brain structures and processes change throughout adolescence and across the life course much of the popular discussion about adolescent brain development has focused on the comparatively late maturation of the frontal lobes, although recent work has broadened the increasing "connectivity" of the brain [7].

Density of the neural connections between the amygdala and cortices that make up the frontal lobes assimilate emotional and cognitive activity in adolescence [7]. They assert that this process continues to progress clearly into adulthood. Adding scientific research to the debate, neuroscience has prompted keen interest in "linking neuromaturation to maturity of judgment suggesting that a conceptual framework that situates brain science in the broader context of adolescent developmental research to facilitate research-to-policy translation is needed" [7].

Child psychologist Laverne Antrobus of London's Tavistock Clinic supports the research findings for she posits it will foster clinicians in guiding young people past the age of 18 to not fall through the gaps in healthcare and education. While there are others who share Antrobus's view, this discourse does not go without its critics. Frank Furedi, a sociologist at the University of Kent disagrees. He stated, "Western culture has infantilized young adults in ways leading to a growing number of men and women in their 20's living at home with their parents...often claimed for economic reasons, but it is not actually for that there is a loss of the aspiration for independence and striking out on your own” [11].

\section{Conclusion}

The change in age for the period of adolescence is currently being discussed in Great Britain and the United States. To date there is not a general consensus across disciplines that this proposed amended age bracket will become the new benchmark for defining the period of adolescence. The Millenials, or Generation Y population would fall into this broadened category. The identified level of stress this cohort exhibits coalesced with their lack of social skills and coping demonstrate a requisite for future research to assess their health needs when affected by celiac disease. Additional discourse is also warranted among the disciplines in addressing the research generated by neuroscience. Whether it is nature or nurture, social or cultural, the question for scholars and researchers remains unanswered. What is the age range for the period of adolescence? It looks like we will have to keep talking.

\section{References}

[1] Arnone, J., Fitzsimons, V., “Adolescents with celiac disease: A literature review of the impact developmental tasks have on adherence with a gluten-free diet," Gastroenterol Nurs, 35(4), 248254, Jul-Aug 2012.

[2] Adolescence. (n.d.) Dictionary.com unabridged. http://dictionary.reference.com/browse/adolescent

[3] Adolescent. (n.d.) Oxford Dictionary. http://www.oxforddictionaries.com/us/definition/american_english /adolescent

[4] Hall, G.S., Adolescence: Its psychology and its relation to physiology, anthropology, sociology, sex, crime, religion and education. New York, NY: Appleton, 1904.

[5] Moshman, D., Adolescents are young adults. not immature brains, Applied Developmental Science, 15(4):171-174, 2011.

[6] Christian, J.L., Philosophy: An introduction to the art of wondering (9th ed.), Belmont, CA: Thomson Wadsworth, 2006, 134.

[7] Johnson, S., Blum, R., Giedd, J., “Adolescent maturity and the brain: The promise and pitfalls of neuroscience research in adolescent health policy,” J Adolesc Health, 45(3), 216-221, Sep 2009.

[8] Cosgrove-Mather, B., "Rethinking the age of adulthood," CBS News, 2003. http://www.cbsnews.com/news/rethinking-the-age-of-adulthood/

[9] Scotti, C., How millenials long road to adulthood is the new normal, FoxBusiness, 2014.

http://www.foxbusiness.com/personal-financial/2014/4/29/howmillenials-long-road-to-adulthood-is-new-normal/

[10] Chung, S.M., "Knowing generation Y: A new generation of nurses in practice,” Br J Nurs, 22(20), 1173-1179, Nov 2013.

[11] Wallis, L., Is 25 the new cut-off point for adulthood?, BBC News Magazine. 2013.

http://www.bbc.com.news/magazine-24173194?print=true 